\title{
A SEARCH FOR SPECTRAL VARIABILITY IN WR1
}

\author{
A. NIEDZIELSKI \\ N. Copernicus University, ul. Chopina 12/18, 87-100 Toruń, Poland
}

\begin{abstract}
Preliminary results of spectroscopic monitoring of the WN5-B star WR1 (HD 4004) are presented, showing evidence for a $0^{\mathrm{d}} .775$ period.
\end{abstract}

\section{Introduction}

WR1 (HD 4004, WN5-B) is a 10.51 (v) magnitude star which spectrum and intrinsic properties are similar to those of WR6 (HD 50896, WN5-B). It is however more distant and substantially reddened. The photometric search for variability (Moffat \& Shara 1986) suggests a period of $6^{\mathrm{d}} .1$ with a net $\mathrm{rms}$ scatter of $0.01 \mathrm{mag}$, while the radial velocity study of Lamontagne (1983) points to a possible period of $8^{\mathrm{d}}$.

\section{Observations}

The observations reported here were taken with the $1.82 \mathrm{~m}$ telescope of $\mathrm{Mt}$. Ekar Observatory equipped with a B\&C spectrograph and CCD detector $(1 \AA /$ pix resolution) in the $5350-5950 \AA$ range (Claudi \& Cremonese 1993). In this range, emission lines of He II $\lambda 5411, \mathrm{C}$ IV $\lambda 5808$ and He I $\lambda 5876$ were observed. We obtained 56 spectra in three consecutive nights, December 4 $-6,1993$. The temporal resolution of spectra during one night was of the order of $15-30$ min during some 4-5 hours, allowing us to look for variability in both long (days) and short (hours) periods.

The overall shape of spectrum in the observed range is well defined, but substantial variability appears in the He II $\lambda 5411$, He I $\lambda 5876$ and C IV $\lambda 5808$ emission lines. The equivalent widths of all lines show a 5-10\% variability

Fig. 1. Rms curve of December 4, 1993, based on 23 spectra.

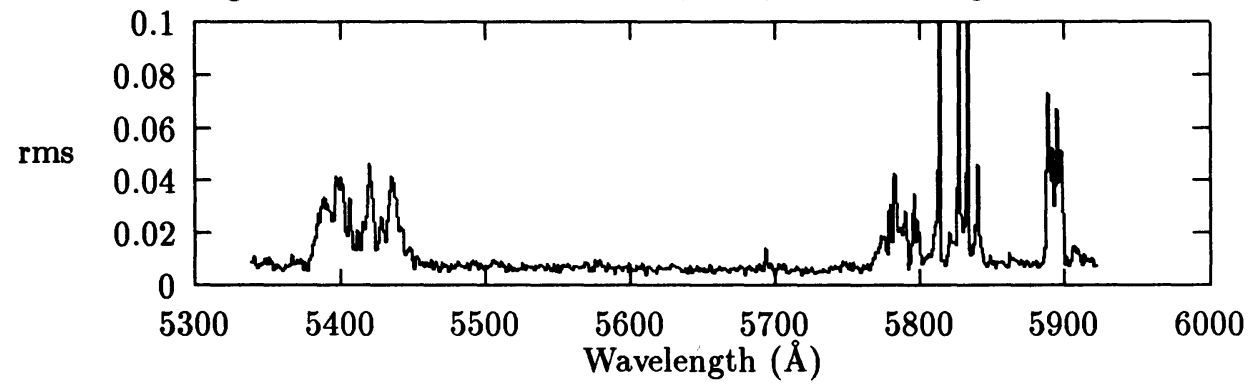


Fig. 2. Skewness of $\lambda 5411$ HeII plotted vs. $0^{d} .775$ period.

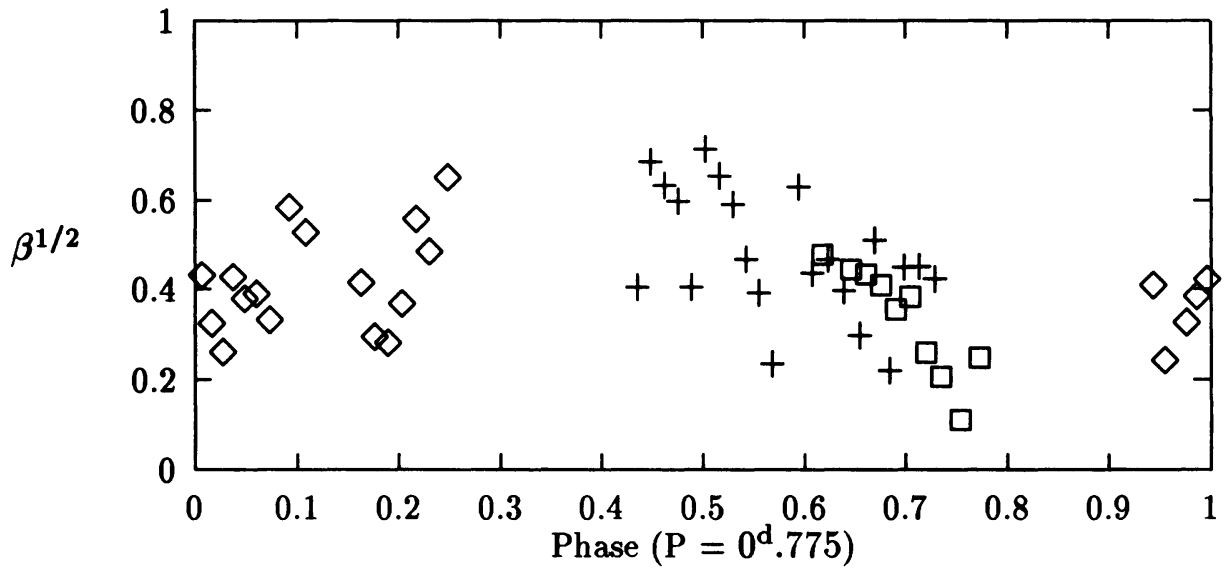

from night to night. Also the radial velocities obtained from the mean wavelengths of emission features vary significantly between nights, as do skewness and kurtosis of line profiles.

The variability in periods shorter than 5 hours is easily seen in equivalent widths, radial velocities, and line profile parameters as well. An example of the rms curve of one night, calculated with the respect to the mean spectrum of the first night is shown in Fig. 1. One can see that rms of the profiles of He II $\lambda 5411$ and CIV $\lambda 5808$ are of the same order as in WR6 as reported by Robert et al. (1992). The amplitude of variations in line profiles seem to change with time: during the following night the rms of He II $\lambda 5411$ reached 0.08 and the next night 0.02 .

The collected data do not allow a complete period analysis. However, a period search performed on all data shows a period of $0^{d} .775$. Our data suggest also a period longer than the time covered by observations. The variations on an hourly scale seem to be random.

\section{Acknowledgements}

This work was supported by the KBN grant No. 221129203 through N. Copernicus University in Torun.

\section{References}

Claudi, R.U., Cremonese, G. 1993, Asiago and Padova Observatories Handbook Helfand, D.J. 1980, PASP 92, 691

Lamontagne, R. 1983, PhD thesis, Université de Montréal

Moffat, A.F.J., Shara, M.M. 1986, AJ 92, 952

Robert, C. et al. 1992, ApJ 397, 277 